\title{
Fleet Sizing in Vehicle Sharing Systems with Service Quality Guarantees
}

\author{
Michal Čáp, Szabolcs Vajna, and Emilio Frazzoli
}

\begin{abstract}
Vehicle sharing system consists of a fleet of vehicles (usually bikes or cars) that can be rented at one station and returned at another station. We study how to achieve guaranteed service availability in such systems. Specifically, we are interested in determining a) the fleet size and initial allocation of vehicles to stations and $b$ ) the minimum capacity of each station needed to guarantee that a) every customer will find an available vehicle at the origin station and b) the customer will find a free parking spot at the destination station. We model the evolution of number of vehicles at each station as a stochastic process and prove that the relevant probabilities in the system can be approximated from above using a computationallytractable decoupled model. This property can be exploited to efficiently determine the size of fleet, initial distribution of vehicles to stations, and station capacities that are sufficient to achieve the desired service level. The applicability of the method is demonstrated by computing the initial vehicle stock and the capacity of each station that would be needed to avoid service failures in Boston's bike sharing system "The Hubway". Our simulation shows that the proposed method is able to find more efficient design parameters than the naive approach and consequently it can achieve the equivalent quality-of-service level with half of the vehicle fleet and half of the parking capacity.
\end{abstract}

\section{INTRODUCTION}

Bike sharing and car sharing systems can act as a sustainable and economically viable alternative to private car ownership in urban environments [9]. A major obstacle to mass adoption of such vehicle sharing systems is the low reliability of service [5]. In many such systems, the users regularly experience service unavailability: Either there are no available vehicles at the origin station when they decide to rent a vehicle or there is not enough parking spots at the destination station when they decide to return a rented vehicle. In order to provide customer experience that is comparable to the comfort of using a privately-owned vehicle, a sharing system should guarantee that a user will always be able to pickup a vehicle at the desired origin location and later return the vehicle at the destination location. Therefore, a critical task of the system operator is to determine a) the size of the vehicle fleet and the allocation of available vehicles to stations at the beginning of the day, b) the capacity of each station, and c) a strategy for future redistribution of vehicles between stations such that no station becomes completely empty and no station becomes completely full throughout the day.

In recent years, researchers have increasingly focused on development of algorithms that support design and operation of vehicle sharing systems [3].

Michal Čáp is with FEL, CTU in Prague and TU Delft.

Szaboles Vajna is with BUTE in Budapest.

Emilio Frazzoli is with ETH Zurich and nuTonomy.
A large portion of existing work focuses on the analysis and synthesis of policies for vehicle rebalancing - since urban transportation patterns are structurally imbalanced (e.g., in the morning, most customers rent vehicles in residential areas and return them in business areas), the vehicles must be continuously moved (rebalanced) from stations with surplus of vehicles to stations with shortage of vehicles. In order to determine efficient rebalancing strategies and to quantify the amount of rebalancing needed in a particular vehicle sharing system, researchers proposed fluidic models [6], queue theoretic models [10], [1] as well as different heuristics [7]. The existing rebalancing models, however, neglect the issue of fleet sizing.

Although the above methods can achieve balanced flows on expectation, in practice, each station must have a sufficient stock of vehicles and sufficient parking capacity to also cover the difference between the incoming and outgoing flows when they deviate from the expectation.

Spieser et al. [8] provide a method for determining a lower bound on a fleet size that is necessary to ensure passenger queue stability, i.e., waiting time should not grow to infinity. In practice, however, one is rather interested in ensuring high-level of availability, i.e., almost all passengers should be served immediately. George and Xia [4] studied the relation between the fleet size and the service availability within a queue-theoretic model. Yet, the analysis is limited to systems with time-invariant demand in asymptotic regime when the number of vehicles in the system goes to infinity. Thus, in order to determine an appropriate fleet-size in a practical system, one typically resorts to simulation-based optimization [11], [12], [2].

The contribution of this paper is therefore a principled treatment of the problem of determining the minimum initial fleet size and parking capacity at each station needed to avoid service failures in the system with desired confidence, given probabilistic time-dependent model of future demand and a fixed rebalancing plan. To evaluate the probability of such a service failure, we model the development of the number of vehicles at each station as a stochastic process. Such a model would be prohibitively expensive to evaluate, but we find that the relevant probabilities can be approximated from above by a computationally-tractable model, where the random variables that represent the number of vehicles at each station are assumed to be independent.

This allows us to optimize over the initial number of vehicles at each station and over the capacity at each station such that system failures are avoided with required confidence. To demonstrate the potential of the proposed method, we use the method to determine the size of the fleet and capacities of 
stations necessary to avoid service failures in Boston's bike sharing system with $99 \%$ confidence. Compared to a naive system design technique, the proposed method achieves the same level of service reliability with less than half of the vehicles and with less than half of the parking capacity.

\section{Problem Statement}

Consider a vehicle sharing system consisting of $k$ stations, with the set of all station labels denoted by $X=\{1, \ldots, k\}$. At time $t=0$, station 1 stores $v_{1}$ vehicles, station 2 stores $v_{2}$ vehicles, etc. A vehicle moves from one station to another when either a) a customer rents a vehicle at one station and returns the vehicle at another stations or b) it is relocated as a part of the rebalancing process.

The customer demand is modeled probabilistically. In particular, the requests to rent vehicle for transportation from station $o$ to station $d$ are assumed to be generated by a nonhomogeneous Poisson point process with intensity $\lambda_{o d}(t)$ at time $t$. The rebalancing vehicles are relocated from station $o$ to station $d$ according to a deterministic rebalancing plan. The plan for rebalancing between stations $o$ and $d$ is represented as a set of time points $\rho_{o d}=\left\{t_{o d}^{1}, t_{o d}^{2}, \ldots\right\}$ prescribing when rebalancing vehicles should depart from station $o$ towards station $d$. Further, let $\rho:=\cup_{o, d \in X} \rho_{o d}$ denote the set of all time points when rebalancing occurs. Whenever a rented or rebalancing vehicle departs from station $o$ destined to station $d$, it will arrive to station $d$ with deterministic delay $\eta_{i j}$.

To successfully pickup a vehicle, the origin station must not be empty. If all pickup requests are served, we say that the system is maintaining full availability. And vice versa, if a customer requests a vehicle and the station is empty, the system is said to fail to maintain full availability. Similarly, each station $i$ has a limited parking capacity $c_{i}$, and a vehicle can be returned at the destination station only when it is not at full capacity. When all arriving vehicles find a free parking spot at their destination station, we say that the system maintains capacity constraints. Conversely, the system fails to maintain capacity constraints if a station is full and a new vehicle arrives to the station. When the system maintains both availability and capacity constraints we say that the system operates failure-free.

As we can see, the failure-free operation of the system depends on the initial stock of vehicles at each station, on the capacity of each station, and on the rebalancing strategy. In this paper, we will assume that the vehicle sharing system employs a particular open-loop rebalancing strategy and we will focus on determining the initial number of vehicles and the capacity of each station that collectively ensure failure-free operation of the system. Since the future demand is generated by a Poisson process, any station can experience arbitrarily high number of travel request with nonzero probability. Therefore, failure-freeness of the system cannot be achieved with certainty. It is, however, reasonable to ask for a system that operates failure free with high probability as stated in the following problem formulation:
Problem 1. Determine the initial number of vehicles $v_{1}, \ldots, v_{k}$ that need to be present at respective stations $1, \ldots, k$ at time $t=0$ and the capacity $c_{1}, \ldots, c_{k}$ of each station $1, \ldots, k$ that ensure that the system will operate failure-free in given future time interval $[0, T]$ with chosen confidence level $(1-z)$ with $z$ representing the maximum allowed probability of failure in the system.

\section{COUPled MODEL}

In this section, we will develop a numerical method for determining the exact probability of failure-free operation of a vehicle sharing system in the time interval of interest $[0, t]$. When developing the model, we neglect the travel delay between stations to maintain analytic tractability, but we show that it can be naturally incorporated in the simpler decoupled model of the next section.

The evolution of the system is modeled as a continuoustime random process $\{V(t)\}_{t \in[0, \infty)}$ over state space $\mathbb{M} \cup$ $\{F\}$. As long as the system operates failure-free, it remains in set $\mathbb{M}$, once it experiences a failure, it falls into an absorbing state denoted by $F$. The failure-free part of state space, defined as $\mathbb{M}:=\mathbb{M}_{1} \times \ldots \times \mathbb{M}_{k}$ with $\mathbb{M}_{i}:=$ $\left\{0, \ldots, c_{i}\right\}$ being the set of all possible numbers of vehicles at station $i$, contains a state for every possible combination of number of vehicles at individual stations. Therefore, if $V(t) \neq F$, then random variable $V(t)=\left(m_{1}, \ldots, m_{k}\right)$ represents the fact that the vehicle sharing system has $m_{1}$ vehicles at station $1, m_{2}$ vehicles at station 2 , etc. To refer to the number of vehicles at a single station, we define random variables $V_{1}(t), \ldots, V_{k}(t)$ as

$$
V_{i}(t):=\left\{\begin{array}{ll}
(V(t))_{i} & \text { if } V(t) \in \mathbb{M} \\
F & \text { if } V(t)=F
\end{array},\right.
$$

where $(\mathbf{x})_{i}$ denotes the $i$-th element of tuple $\mathbf{x}$. Observe that the random variables $V_{1}(t), \ldots, V_{k}(t)$ are not independent. They are coupled, e.g., by the constraint that the number of vehicles in the system is constant, i.e., for every time point $t$ we have $\sum_{i=1, \ldots, k} V_{i}(t)=\sum_{i=1, \ldots, k} V_{i}(0)=$ const. Therefore, the model described in this section will be referred to as coupled model.

The probability that the system has been failure-free in time interval $[0, t]$ and there is $m_{1}$ vehicles at station $1, m_{2}$ vehicles at station 2 , etc. at time $t$ is denoted by $p_{\mathbf{m}}(t)$, where $\mathbf{m}=\left(m_{1}, \ldots, m_{k}\right)$. We define $p_{\mathbf{m}}(t):=\mathbb{P}(V(t)=\mathbf{m})$ for any $\mathbf{m} \in \mathbb{M}$ and $p_{\mathbf{m}}(t):=0$ otherwise.

The collection of probabilities $p_{\mathbf{m}}\left(t^{\prime}\right)$ for all failure-free states $\mathbf{m} \in \mathbb{M}$ and all time points $t^{\prime} \in[0, t]$ can be expressed as a time-dependent $k$-dimensional tensor $\mathbf{P}\left(t^{\prime}\right)=$ $\left\{p_{\mathbf{m}}\left(t^{\prime}\right)\right\}_{\mathbf{m} \in \mathbb{M}} \in[0,1]^{\mathbb{M}_{1} \times \ldots \times \mathbb{M}_{k}}$. The probability that the system is failure-free in time interval $[0, t]$ is $p_{N}(t):=$ $\mathbb{P}(V(t) \in \mathbb{M})$. Similarly, the probability that the system has experienced a failure in time interval $[0, t]$ is $p_{F}(t):=$ $\mathbb{P}(V(t)=F)$. Observe that we have $p_{N}(t)=\sum_{\mathbf{m} \in \mathbb{M}} p_{\mathbf{m}}(t)$ and $p_{F}(t)=1-p_{N}(t)$.

Further, we write $p_{F}\left(t ;\left(v_{1}, \ldots, v_{k}\right),\left(c_{1}, \ldots, c_{k}\right)\right)$ to denote the probability $\mathbb{P}(V(t)=F)$ when the initial numbers 
of vehicles and capacities at stations $1, \ldots, k$ are fixed to $v_{1}, \ldots, v_{k}$ and $c_{1}, \ldots, c_{k}$ respectively.

In order to be able to concisely express the dynamics of the modeled process, we need to introduce some additional notation. Firstly, the following shorthand notation is used to refer to subsets of $\mathbb{M}$ consisting of states satisfying constraints on the number of vehicles at some of the stations:

$$
\begin{array}{ll}
\mathbb{M}^{i=j}:= & \left\{\mathbf{m} \mid \mathbf{m}=\left(m_{1}, \ldots, m_{k}\right) \in \mathbb{M} \text { and } m_{i}=j\right\}, \\
\mathbb{M}^{i>j}:= & \left\{\mathbf{m} \mid \mathbf{m}=\left(m_{1}, \ldots, m_{k}\right) \in \mathbb{M} \text { and } m_{i}>j\right\}, \\
\mathbb{M}^{i<j}:= & \left\{\mathbf{m} \mid \mathbf{m}=\left(m_{1}, \ldots, m_{k}\right) \in \mathbb{M} \text { and } m_{i}<j\right\} .
\end{array}
$$

Further, constraints may be chained, e.g., $\mathbb{M}^{i_{1}=j_{1}, i_{2}<j_{2}}$ will be a shorthand for $\mathbb{M}^{i_{1}=j_{1}} \cap \mathbb{M}^{i_{2}<j_{2}}$.

The family of functions $T_{o d}: \mathbb{Z}^{k} \rightarrow \mathbb{Z}^{k}$ for $o, d \in X \times X$ encodes the effect of a relocation of single vehicle from station $o$ to station $d$ on the number of vehicles at each station. More specifically, $T_{o d}(\mathbf{m})$ represents the number of vehicles at each station when the system was in state $\mathbf{m}$ and a single vehicle relocated from station $o$ to station $d$ :

$$
\begin{gathered}
T_{o d}: \mathbf{m}=\left(m_{1}, \ldots, m_{k}\right) \mapsto \mathbf{m}^{\prime}=\left(m_{1}^{\prime}, \ldots, m_{k}^{\prime}\right), \\
\text { where } m_{i}^{\prime}= \begin{cases}m_{i}-1 & \text { if } i=o \\
m_{i}+1 & \text { if } i=d \\
m_{i} & \text { otherwise. }\end{cases}
\end{gathered}
$$

As a special case, if the origin and destination stations are the same, we define $T_{i i}(\mathbf{m})=\mathbf{m}$. Note that transformation $T$ does not account for capacity and availability constraints. Further, let $T^{-1}$ denote the inverse of $T$, i.e., $\left(T_{o d}\right)^{-1}(\mathbf{m}=$ $\left.\left(m_{1}, \ldots, m_{n}\right)\right)=\mathbf{m}^{\prime}$ such that $T_{o d}\left(\mathbf{m}^{\prime}\right)=\mathbf{m}$.

We are now in position to describe the dynamics of the system. Suppose that initially, at time $t=0$, the stations $1, \ldots k$ have $v_{1}, \ldots, v_{k}$ vehicles respectively. Then, the initial condition of $p_{\mathbf{m}}$ is $p_{\mathbf{m}}(0)=1$ for $\mathbf{m}=\left(v_{1}, \ldots, v_{k}\right)$ and $p_{\mathbf{m}}(0)=0$ for every other value of $\mathbf{m}$. The system is initially failure-free and thus we have $p_{F}(0)=0$.

The functions $\left\{p_{\mathbf{m}}\right\}_{\mathbf{m} \in \mathbb{M}}$ and $p_{F}$ are piecewise differentiable on $\mathbb{R}_{\geq 0}$. At time points $t_{1}, t_{2}, \ldots \in \rho$, vehicle is rebalanced between stations and thus the functions $\left\{p_{\mathbf{m}}\right\}_{\mathbf{m} \in \mathbb{M}}$ and $p_{F}$ contain a jump discontinuity. Formally, for every $o, d \in X$, and $t \in \rho_{\text {od }}$ we have

$$
\begin{aligned}
p_{\mathbf{m}}(t) & =\lim _{t^{\prime} \rightarrow t^{-}} p_{T_{o d}^{-1}(\mathbf{m})}\left(t^{\prime}\right) \quad \text { and } \\
p_{F}(t) & =\lim _{t^{\prime} \rightarrow t^{-}} p_{F}\left(t^{\prime}\right)+\sum_{\mathbf{m} \in \mathbb{M}^{o=0} \cup \mathbb{M}^{d=c_{i}}} p_{\mathbf{m}}\left(t^{\prime}\right) .
\end{aligned}
$$

Everywhere else, i.e., at any $t \in \mathbb{R}_{\geq 0} \backslash \rho$, the functions $\left\{p_{\mathbf{m}}\right\}_{\mathbf{m} \in \mathbb{M}}$ and $p_{F}$ are differentiable and their time evolution is governed by the following system of differential equations. For every $\mathbf{m} \in \mathbb{M}$ and $t \in \mathbb{R}_{\geq 0} \backslash \rho$,

$$
\begin{aligned}
& \dot{p}_{\mathbf{m}}(t)=\sum_{o, d \in X} \lambda_{o d}(t) \cdot p_{T_{o d}^{-1}(\mathbf{m})}(t)-p_{\mathbf{m}}(t) \cdot \sum_{o, d \in X} \lambda_{o d}(t) \text { and } \\
& \dot{p}_{F}(t)=\sum_{o, d \in X} \lambda_{o d}(t) \sum_{\mathbf{m} \in \mathbb{M}^{o=0} \cup \mathbb{M}^{d=c_{i}}} p_{\mathbf{m}}(t) .
\end{aligned}
$$

Proposition 2. This construction preserves probability, that $i s$, for every $t \in \mathbb{R}_{\geq 0}$ we have $\sum_{m \in \mathbb{M}} p_{\mathbf{m}}(t)+p_{F}(t)=1$.

Proof. See Appendix for formal proof.

\section{DeCOUPled Method}

The evaluation of the coupled model requires numerical integration over all elements from $\mathbb{M}$. Since the number of elements in $\mathbb{M}$ grows exponentially in the number of stations, this method becomes computationally intractable when applied to systems with more than a few stations.

For larger systems, we propose to use an alternative decoupled model, where the individual stations are modeled by independent stochastic processes. In contrast to the coupled model, where the relocation of vehicles between every pair of stations $o$ and $d$ is governed by a single Poisson process with intensity $\lambda_{o d}$, the relocation of vehicles in the decoupled model is governed by a departure process and an independent arrival process, both with intensity $\lambda_{o d}$. The departure process from $o$ to $d$ generates time points when customers request to rent vehicles from station $o$ towards station $d$, while the arrival process generates time points when vehicles rented from station $o$ arrive to station $d$. Note that in such a setting, the physical correspondence between vehicles from the departure process and the vehicles from the arrival process is lost. In fact, it can happen that at some time point $t$, we have more vehicles that have arrived at some station $i$ than there are vehicles that have departed towards station $i$. Analogously to the coupled model, when a vehicle is requested from an empty station, the station experiences an availability failure. When a vehicle arrives to a full station, the station experiences capacity failure.

Decoupling the departure and arrival processes enables us to reason about the evolution of the number of vehicles at each station separately and consequently evaluate the probability of failure more efficiently. In the decoupled model, we model the evolution of the system as a collection of $k$ random processes. The evolution of the stock of vehicles at station $i$ is a continuous-time random process $\left\{\bar{V}_{i}(t)\right\}$ over state space $\mathbb{M}_{i} \cup\{F\}$. As long as the station operates failure-free, it remains in the set $\mathbb{M}_{i}:=\left\{1, \ldots, c_{i}\right\}$, once the station experiences a failure, it falls into an absorbing state $F$. Therefore, if $\bar{V}_{i}(t) \neq F$, then $\bar{V}_{i}(t)=j$ represents the fact that the station $i$ currently has $j$ vehicles.

The probability that station $i$ at time $t$ has $j$ vehicles and has not experienced a failure yet is denoted $q_{i}^{j}(t)$. We define $q_{i}^{j}(t):=P\left(\bar{V}_{i}(t)=j\right)$ for $0 \leq j \leq c_{i}$ and $q_{i}^{j}(t):=0$ for other values of $j$. The probability that station $i$ has experienced a failure in time interval $[0, t]$ is $q_{i}^{F}(t):=P\left(\bar{V}_{i}(t)=F\right)$.

Further, we write $q_{i}^{F}(t ; v, c)$ to denote the probability $\mathbb{P}\left(\bar{V}_{i}(t)=F\right)$ when the initial number of vehicles at station $i$ is $v$ and its capacity is $c$. The initial condition for $q_{i}^{j}$ for every $i \in X$ is $q_{i}^{j}(0)=1$ for $j=v_{i}$, where $v_{i}$ is the number of vehicles at station $i$ at time 0 , and $q_{i}^{j}(0)=0$ for other values of $j$. Initially, each station is failure-free and thus we have $q_{i}^{F}(0)=0, \forall i \in X$. Let $\lambda_{i}^{a}(t):=\sum_{o \in X \backslash\{i\}} \lambda_{o i}(t)$ be the total intensity of all arriving vehicle flows to station $i$ and $\lambda_{i}^{d}:=\sum_{d \in X \backslash\{i\}} \lambda_{i d}(t)$ be the total intensity of all departing vehicle flows from station $i$. Analogously, let $\rho_{i}^{a}:=$ $\cup_{o \in X \backslash\{i\}} \rho_{o i}$ be the set of time points when rebalancing 
vehicles are scheduled to arrive to station $i$ and $\rho_{i}^{d}:=$ $\cup_{d \in X \backslash\{i\}} \rho_{i d}$ be the set of time points when rebalancing vehicles are scheduled to depart from station $i$. The functions $\left\{q_{i}^{j}\right\}_{j \in \mathbb{M}_{i}}$ and $q_{i}^{F}$ for a particular station $i$ are piecewise differentiable with a discontinuous jump at every time point $t \in \rho_{i}^{a}$ as follows

$$
q_{i}^{j}(t)=\lim _{t^{\prime} \rightarrow t^{-}} q_{i}^{j-1}\left(t^{\prime}\right) ; \quad q_{i}^{F}(t)=\lim _{t^{\prime} \rightarrow t^{-}} q_{i}^{F}\left(t^{\prime}\right)+q_{i}^{c_{i}}\left(t^{\prime}\right)
$$

and at every time point $t \in \rho_{i}^{d}$ as follows

$$
q_{i}^{j}(t)=\lim _{t^{\prime} \rightarrow t^{-}} q_{i}^{j+1}\left(t^{\prime}\right) ; \quad q_{i}^{F}(t)=\lim _{t^{\prime} \rightarrow t^{-}} q_{i}^{F}\left(t^{\prime}\right)+q_{i}^{0}\left(t^{\prime}\right) .
$$

Everywhere else, i.e., for all $t \in \mathbb{R}_{\geq 0} \backslash\left(\rho_{i}^{a} \cup \rho_{i}^{d}\right)$, the functions $\left\{q_{i}^{j}\right\}_{j \in \mathbb{M}_{i}}$ and $q_{i}^{F}$ are differentiable and their evolution is governed by the following system of differential equations:

$$
\begin{aligned}
\dot{q}_{i}^{j}(t) & =\lambda_{i}^{a} \cdot q_{i}^{j-1}(t)-\left(\lambda_{i}^{a}+\lambda_{i}^{d}\right) \cdot q_{i}^{j}(t)+\lambda_{i}^{d} \cdot q_{i}^{j+1}(t), \\
\dot{q}_{i}^{F}(t) & =\lambda_{i}^{a}(t) \cdot q_{i}^{c_{i}}+\lambda_{i}^{d}(t) \cdot q_{i}^{0}(t) .
\end{aligned}
$$

If we denote $\mathbf{q}_{i}(t)=\left[q_{i}^{0}(t), \ldots, q_{i}^{c_{i}}(t)\right]$, then the behavior of the function $\mathbf{q}_{i}$ at discontinuous jumps can be conveniently expressed as right or left "shift" of the state vector:

$$
\begin{aligned}
\forall t \in \rho_{i}^{a}: \mathbf{q}_{i}(t) & =\lim _{t^{\prime} \rightarrow t^{-}}\left[0, q_{i}^{0}\left(t^{\prime}\right), \ldots, q_{i}^{c_{i-1}}\left(t^{\prime}\right)\right] \\
& =\left[0, q_{0}, \ldots, q_{c_{i}-1}\right], \text { where } q=\lim _{t^{\prime} \rightarrow t^{-}} \mathbf{q}_{i}\left(t^{\prime}\right) \\
\forall t \in \rho_{i}^{d}: \mathbf{q}_{i}(t) & =\lim _{t^{\prime} \rightarrow t^{-}}\left[q_{i}^{1}\left(t^{\prime}\right), \ldots, q_{i}^{c_{i}}\left(t^{\prime}\right), 0\right] \\
& =\left[q_{1}, \ldots, q_{c_{i}}, 0\right], \text { where } q=\lim _{t^{\prime} \rightarrow t^{-}} \mathbf{q}_{i}\left(t^{\prime}\right) .
\end{aligned}
$$

The above system of differential equations can be expressed in a matrix form $\dot{\mathbf{q}}_{i}(t)=Q_{i}(t) \cdot \mathbf{q}_{i}(t)$, with $Q_{i}(t)=$

$$
\left[\begin{array}{ccccc}
-\left(\lambda_{i}^{d}+\lambda_{i}^{a}\right) & \lambda_{i}^{d} & 0 & 0 & \cdots \\
\lambda_{i}^{a} & -\left(\lambda_{i}^{d}+\lambda_{i}^{a}\right) & \lambda_{i}^{d} & 0 & \cdots \\
0 & \lambda_{i}^{a} & -\left(\lambda_{i}^{d}+\lambda_{i}^{a}\right) & \lambda_{i}^{d} & \cdots \\
0 & 0 & \lambda_{i}^{a} & -\left(\lambda_{i}^{d}+\lambda_{i}^{a}\right) & \cdots \\
\vdots & \vdots & \vdots & \vdots & \ddots
\end{array}\right] .
$$

(the argument $t$ of functions $\lambda_{i}^{d}$ and $\lambda_{i}^{a}$ was dropped for brevity)

In result, the vector function $\mathbf{q}_{i}$ can be efficiently evaluated using a numerical computation software, and the probability of failure at a station can be obtained as $q_{i}^{F}(t):=1-$ $\sum_{j \in \mathbb{M}_{i}} q_{i}^{j}(t)$.

We will now show that the decoupled model of the system can be used to obtain an upper-bounding approximation of the failure probability in the coupled model.

Theorem 3. The probability that a particular station $i$ has $j$ vehicles in the decoupled model upper-bounds the probability that the same station $i$ has $j$ vehicles in the coupled model, i.e., it holds that $P\left(V_{i}(t)=j\right) \leq P\left(\bar{V}_{i}(t)=j\right)$ for all $i \in X, j \in \mathbb{M}_{i}$, and $t \geq 0$.

\section{Proof. See Appendix for formal proof.}

For intuitive justification of this property, consider a system with three stations $X=\{a, b, c\}$ in which at time $t$ we have an attempt to rent a vehicle from station $a$ to station $b$. In the decoupled model, the arrival and departure processes are seen as independent and thus the probability that station $b$ has, e.g., two vehicles after the event, $P\left(\bar{V}_{b}(t)=2\right)$, is defined to be equal to the probability that it had one vehicle before the event, $P\left(\lim _{t^{\prime} \rightarrow t^{-}} \bar{V}_{b}\left(t^{\prime}\right)=1\right)$. Notice that this probability does not depend on the state of station $a$. In the coupled model, however, the relocation will not occur if station $a$ is empty, which would be represented as $P\left(\lim _{t^{\prime} \rightarrow t^{-}} V_{b}\left(t^{\prime}\right)=\right.$ 1 and $\left.V_{a}\left(t^{\prime}\right) \neq 0\right)$ or equivalently $P\left(\lim _{t^{\prime} \rightarrow t^{-}} V_{b}\left(t^{\prime}\right)=1\right)$ - $P\left(\lim _{t^{\prime} \rightarrow t^{-}} V_{a}\left(t^{\prime}\right)=0\right.$ and $\left.V_{b}\left(t^{\prime}\right)=1\right)$. Because the decoupled model does not account for the second term, the probability $P\left(\bar{V}_{i}(t)=j\right)$ consistently overestimates the probability $P\left(V_{i}(t)=j\right)$. Analogously, the probability that station $c$ has, e.g., three vehicles after the event $P\left(\bar{V}_{c}(t)=3\right)$, is in the decoupled model equal to $P\left(\lim _{t^{\prime} \rightarrow t^{-}} \bar{V}_{c}\left(t^{\prime}\right)=3\right)$, because there are no vehicles arriving to or departing from station $c$. In the coupled model, however, if station $a$ is empty or if station $b$ is full, the entire system will fail and the probability of station $c$ having three vehicles would be defined as $P\left(\lim _{t^{\prime} \rightarrow t^{-}} V_{c}\left(t^{\prime}\right)=3\right.$ and $V_{a}\left(t^{\prime}\right) \neq 0$ and $\left.V_{b}\left(t^{\prime}\right) \neq c_{b}\right)$ or equivalently as $P\left(\lim _{t^{\prime} \rightarrow t^{-}} V_{c}\left(t^{\prime}\right)=3\right) \quad-$ $P\left(\lim _{t^{\prime} \rightarrow t^{-}} V_{c}\left(t^{\prime}\right)=3\right.$ and $\left(V_{a}\left(t^{\prime}\right)=0\right.$ or $\left.\left.V_{c}\left(t^{\prime}\right)=c_{d}\right)\right)$. Again, the second term is not accounted for in the decoupled model and thus the probability $P\left(\bar{V}_{c}(t)=3\right)$ overestimates probability $P\left(V_{c}(t)=3\right)$. Similar reasoning can be used to show that the sum of station failure probabilities in the decoupled model also overestimates the probability of system failure in the coupled model.

Corollary 4. The probability of failure in the coupled model is upper bounded by the sum of probabilities of failure at every station in the decoupled model, that is, $P(V(t)=$ $F) \leq \sum_{i \in X} P\left(\bar{V}_{i}(t)=F\right)$.

Proof. See Appendix for formal proof.

Figure 11 illustrates the discrepancy between the failure probability in decoupled model $\sum_{i \in x} q_{i}^{F}$ and the failure probability in coupled model $p_{F}$ for an example vehicle sharing system of practical size. We can see that quantity $\sum_{i \in x} q_{i}^{F}$ is a reasonable approximation of $p_{F}$ for small values of $p_{F}$. Recall that our goal is to determine if the probability of failure exceeds some small threshold value $z$, e.g., $z=1 \%$, at time point $T$, and thus we can make use of this property and use $\sum_{i \in x} q_{i}^{F}$ as a substitute of $p_{F}$.

The previous analysis assumed instantaneous relocation of vehicles between stations, which allowed us to formulate the coupled model as a memory-less random process and consequently maintain analytic tractability. In the decoupled model, however, the travel delay can be incorporated in a relatively straightforward way by appropriately shifting the intensity of arrival process relative to the intensity of the respective departure processes. That is, when the intensity of the departure process from station $o$ to station $d$ at time $t$ is $\lambda_{o d}(t)$, then the intensity of the arrival process from station $o$ to station $d$ at time $t$ is $\lambda_{o d}^{a}(t):=\lambda_{o d}\left(t-\eta_{o d}\right)$, where $\eta_{o d}$ is the travel time from station $o$ to station $d$. Analogously, the total intensity of arrivals to station $i$ at time $t$ would 

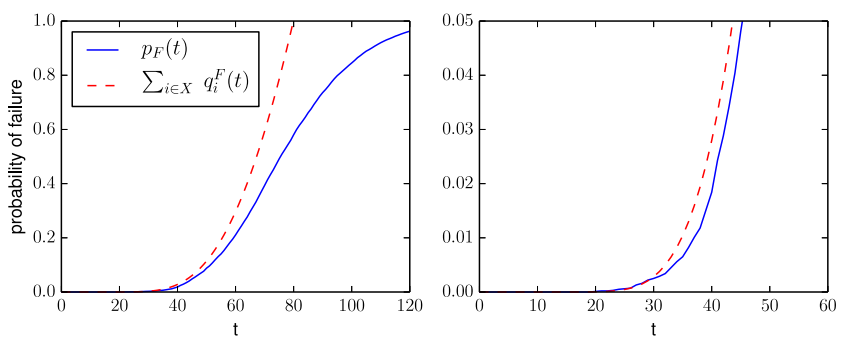

Fig. 1. The illustration of the upper-bounding approximation of the probability of failure obtained from the decoupled model and the exact probability of failure in the coupled model. We compare the two models using a vehicle sharing system with $k=50$ stations, where each station $i$ has initially $v_{i}=50$ vehicles with maximum capacity $c_{i}=100$ vehicles The intensity of travel demand between every two stations $o, d \in X$ is $\lambda_{o d}=0.05$. The solid line shows the probability of failure in the coupled model $p_{F}(t)$ obtained by averaging 20000 simulated realizations of the random process, the dashed line shows the upper bound on $p_{F}(t)$ obtained by evaluating the decoupled model. The right plot shows the behavior of the two functions for small values of $p_{F}(t) \leq 5 \%$.

be defined as $\lambda_{i}(t):=\sum_{o \in X} \lambda_{o i}\left(t-\eta_{o i}\right)$ and the set of timepoints when rebalancing vehicles arrive to station $i$ is $\rho_{i}^{a}:=\cup_{o \in X}\left\{t+\eta_{o i} \mid t \in \rho_{o i}\right\}$.

\section{Optimizing System PARAmeters}

Now we return back to Problem 1, which asks for the number of vehicles needed initially at each station and for the capacity of each station to ensure that the system will be failure free in time interval $[0, T]$ with given given confidence level $1-z$. Ideally, we would like to find the minimal number of vehicles and the smallest number of parking spaces that suffice to satisfy our quality of service constraint, i.e., we would like to solve the following optimization problem

$$
\begin{aligned}
\left\{\left(v_{i}^{\star}, c_{i}^{\star}\right)\right\}_{i}=\underset{\left\{\left(v_{i}, c_{i}\right)\right\}_{i} \in \mathbb{N}^{2 k}}{\operatorname{argmin}} \sum_{i} v_{i}+\sum_{i} c_{i} \\
\text { subject to } p_{F}\left(T ;\left\{\left(v_{i}, c_{i}\right)\right\}_{i}\right) \leq z .
\end{aligned}
$$

The evaluation of $p_{F}(T)$ is, however, computationally intractable for vehicle systems of practical sizes. Instead, we can exploit Corollary 4 and the observation that for small failure threshold $z$, the failure probability in the coupled model can be bounded from above by the sum of failure probabilities at each station to replace term $p_{F}\left(T ;\left\{\left(v_{i}, c_{i}\right)\right\}_{i}\right)$ with $\sum_{i} q_{i}^{F}\left(T ; v_{i}, c_{i}\right)$, which yields the optimization problem

$$
\begin{aligned}
&\left\{\left(v_{i}^{\prime}, c_{i}^{\prime}\right)\right\}_{i}=\underset{\left\{\left(v_{i}, c_{i}\right)\right\}_{i} \in \mathbb{N}^{2 k}}{\operatorname{argmin}} \sum_{i} v_{i}+\sum_{i} c_{i} \\
& \text { subject to } \sum_{i} q_{i}^{F}\left(T ; v_{i}, c_{i}\right) \leq z .
\end{aligned}
$$

Note, that since $p_{F}\left(T ;\left\{\left(v_{i}, c_{i}\right)\right\}_{i}\right) \leq \sum_{i} q_{i}^{F}\left(T ; v_{i}, c_{i}\right)$, a solution $\left\{\left(v_{i}^{\prime}, c_{i}^{\prime}\right)\right\}$ to the optimization problem in Equation 2 is also a feasible, albeit possibly suboptimal, solution for the problem in Equation 11. The constraint function can now be efficiently evaluated, but the formulation in Equation 2 still represents a large-scale non-linear integer optimization problem, which are notoriously hard to solve using existing methods.
Observe that each individual term $q_{i}^{F}\left(T ; v_{i}, c_{i}\right)$ in the constraint function represents the probability that the system failure occurs at station $i$. From this perspective, the optimization problem must assign a budget of probabilities of failure to the individual stations in a way that minimizes the total number of vehicles and the total parking capacity used. This is again a challenging combinatorial optimization problem. The complexity can be circumvented by picking some desired partitioning of system failure probability $z$ to failure probabilities at individual stations $z_{1}, \ldots z_{k}$ such that $z=\sum_{i} z_{i}$. For simplicity we will use uniform partitioning that assigns $z_{i}=z / k$ for every station $i$. For any such fixed partitioning, we can find the minimum number of vehicles $v_{i}$ and minimum parking capacity $c_{i}$ for each station $i$ as

$$
v_{i}, c_{i}=\underset{v, c \in \mathbb{N}}{\operatorname{argmin}} v+c \text { subject to } q_{i}^{F}(T ; v, c) \leq z_{i} .
$$

The above can be solved, e.g., by exhaustive enumeration of all value combination for parameters $v$ and $c$. To improve performance, we find an approximate optimal values for each station by optimizing by coordinates. Recall that the evolution of $q_{i}^{F}$ is governed by equation $\dot{q}_{i}^{F}(t)=\lambda_{i}^{a}(t)$. $q_{i}^{c_{i}}+\lambda_{i}^{d}(t) \cdot q_{i}^{0}(t)$ with initial condition $q_{i}^{F}(0)=0$. The two terms in the differential equation in fact represent the probability of capacity failure and availability failure respectively. The probability of availability failure decreases with increasing number of vehicles and the probability of capacity failure decreases with increasing parking capacity at the station. Therefore, we start by finding the minimal number of vehicles that suffices to cap the probability of availability failure by $z_{i} / 2$ as

$$
v_{i}=\underset{v \in \mathbb{N}}{\operatorname{argmin}} v \text { subject to } q_{i}^{F}(T ; v, \infty) \leq \frac{z_{i}}{2} .
$$

Then, for fixed $v_{i}$, we find the minimal parking capacity at station $i$ that ensures that the probability of system failure at the station is no more than $z_{i}$ as

$$
c_{i}=\underset{c \in \mathbb{N}}{\operatorname{argmin}} c \text { subject to } q_{i}^{F}\left(T ; v_{i}, c\right) \leq z_{i} .
$$

Since both $q_{i}^{F}(T ; v, \infty)$ and $q_{i}^{F}\left(T ; v_{i}, c\right)$ are monotonically non-increasing in $v$ and $c$ respectively, these optimization problems can be efficiently solved, e.g., by the method of bisection. The series of steps described above can find the system parameters $\left\{\left(v_{i}, c_{i}\right)\right\}$ that represent a feasible solution to problem in Equation 1.

\section{CAse Study: HubWay in Boston}

In this section, we demonstrate the applicability of the proposed technique in the context of Boston's bike sharing system called "The Hubway". This system consists of roughly 150 stations scattered over the larger Boson area as shown in Figure 2. The Hubway users often face availability or capacity failures in the system. As a hypothetical exercise, we use our method to determine what fleet size and parking capacity would be sufficient to avoid such failure events.

The Hubway releases anonymized historical data about all bike rentals in the system. We use this dataset to estimate the 


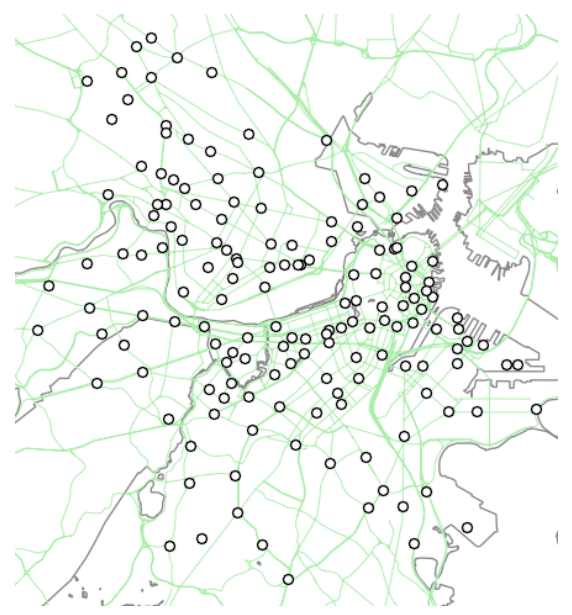

Fig. 2. The stations of Boston's bike sharing system "The Hubway".
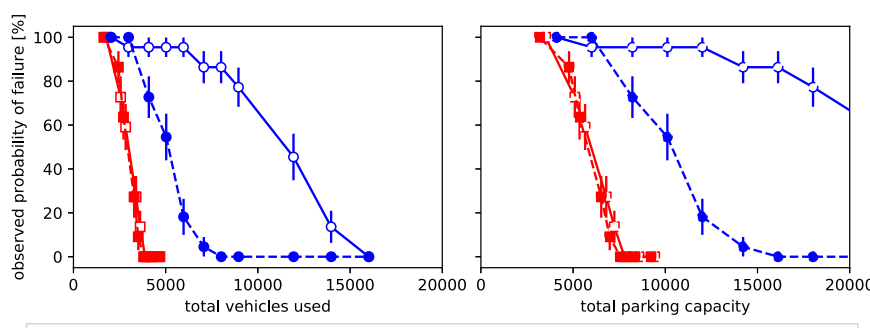

-† proposed (no reb.)

$-\%$ - proposed (w reb.) $\quad-\$-$ baseline (no reb.)

Fig. 3. The plot shows the relation between probability of failure during a day (24h period) and the total fleet size (left) and total parking capacity used (right) when 1) each station has the same parking capacity and initially it is half filled with bikes and half empty (baseline) and 2) when the initial number of bikes and parking capacity at each station is computed using the proposed method (proposed). The dashed line shows the relation when rebalancing of bikes is used and the solid line shows the relation for systems without rebalancing.

parameters of the system model and to evaluate performance of a particular system design using historical rental data. For our experiment, we extracted a data set of all bike rental records realized during working days of May 2016. Then, we used the data set to estimate the intensities of demand generating processes $\left\{\lambda_{o d}\right\}$ in our model and to estimate the travel times $\left\{\eta_{o d}\right\}$.

As expected, the structure of the demand is imbalanced. One can either ignore the imbalance or try to counteract it by relocating vehicles from stations where surplus of bikes is expected to stations where shortage of bikes is expected. In our experiment, we consider both systems that do not use rebalancing and systems where the vehicles are rebalanced during the day. For systems with rebalancing, we find an a priori rebalancing strategy that on expectation balances the rate of bike arrivals and the rate of bike departures at each station using the method proposed in [8].

Next, we need to determine the initial number of vehicles at each station and the parking capacity at each station that suffice to avoid service failures with desired confidence. We compare the optimization-based method proposed in Section $\mathrm{V}$ with a baseline method that assigns equal parking capacity to all stations and initially fills half of the parking spaces at each station with bikes. Then, we evaluate how a system with given design parameters would perform if it had to face a particular sequence of rentals during a day. For performance evaluation, we use 22 historical rental sequences that correspond to rental demand during each working day of May 2016. For every rental recorded in the sequence and for every rebalancing request dictated by the rebalancing plan (if rebalancing is used), we relocate a single vehicle from origin of the rental request to the destination with appropriate travel delay. If the origin station is empty or the destination station is full during any of such relocation, we record that the system failed on that day.

The plots in Figure 3 show the probability of system failure during one day as a function of fleet size and total parking capacity for the proposed method and the baseline method, both with and without rebalancing. We can observe that the proposed method assigns the vehicles and parking capacity to stations more efficiently than the baseline approach. In result, by applying the proposed approach, one can avoid service failures in the system with overwhelming probability ( $\geq 99 \%$ ) using only half of the vehicle fleet and half of the parking capacity compared to what would be needed in a system designed using a baseline approach.

\section{CONCLUSION}

We studied if it is possible to design a vehicle sharing system with service availability guarantees, i.e., a system where all passengers are served with given, arbitrarily high probability. In particular, we propose a systematic method for determining the appropriate system design parameters, namely the initial number of vehicles at each station and the parking capacity at each station, that are sufficient to guarantee that a) all attempts to pickup a vehicle are successful and b) all attempts to return a vehicle are successful, with a given minimum confidence level. In order to determine the probability of a service failure for a particular system design, one has to reason about stochastic vehicle relocations and their effect on the stock of vehicles at each station. However, the model of such system is a random process with exponentially large state space that is prohibitively expensive to evaluate.

Our main contribution is a theoretical analysis leading to an insight that the relevant phenomena in such a system can be sufficiently grasped in a simpler, decoupled stochastic model, where each station is analyzed separately. We prove that the probability of failure in the decoupled model is an upper bounding approximation of the failure probability in the coupled model. Such a finding can be used to efficiently determine the system design parameters that guarantee the desired quality of service in the system. We demonstrate the applicability of the method by computing the system design parameters for a bike sharing system in Boston, and show that the proposed method can achieve the same quality of service as baseline method with only half of the bike fleet and half of the parking capacity. 
Acknowledgments: We would like to thank Slava Kungurtsev for assistance with some of the mathematical problems that the theoretical analysis in this paper hinges on. This research was supported by the Czech Science Foundation (grant No. 18-23623S) and OP VVV MEYS funded project CZ.02.1.01/0.0/0.0/16_019/0000765 "Research Center for Informatics". Access to computing and storage facilities owned by parties and projects contributing to the National Grid Infrastructure MetaCentrum, provided under the program "Projects of Large Infrastructure for Research, Development, and Innovations" (LM2010005), is greatly appreciated.

\section{REFERENCES}

[1] G. C. Calafiore, C. Novara, F. Portigliotti, and A. Rizzo. A flow optimization approach for the rebalancing of mobility on demand systems. In 2017 IEEE 56th Annual Conference on Decision and Control (CDC), pages 5684-5689, December 2017.

[2] Daniel J. Fagnant and Kara M. Kockelman. Dynamic ride-sharing and fleet sizing for a system of shared autonomous vehicles in Austin, Texas. Transportation, 45(1):143-158, January 2018.

[3] D. Gavalas, C. Konstantopoulos, and G. Pantziou. Chapter 13 - Design and management of vehicle-sharing systems: A survey of algorithmic approaches. In Mohammad S. Obaidat and Petros Nicopolitidis, editors, Smart Cities and Homes, pages 261-289. Morgan Kaufmann, Boston, 2016.

[4] David K. George and Cathy H. Xia. Fleet-sizing and service availability for a vehicle rental system via closed queueing networks. European Journal of Operational Research, 211(1):198-207, May 2011.

[5] Katzev Richard. Car Sharing: A New Approach to Urban Transportation Problems. Analyses of Social Issues and Public Policy, 3(1):6586, December 2003.

[6] Marco Pavone, Stephen L Smith, Emilio Frazzoli, and Daniela Rus. Robotic load balancing for mobility-on-demand systems. The International Journal of Robotics Research, 31(7):839-854, June 2012.

[7] Kevin Spieser, Samitha Samaranayake, Wolfgang Gruel, and Emilio Frazzoli. Shared-Vehicle Mobility-on-Demand Systems: A Fleet Operator's Guide to Rebalancing Empty Vehicles. In Transportation Research Board 95th Annual Meeting. Transportation Research Board, 2016.

[8] Kevin Spieser, Kyle Treleaven, Rick Zhang, Emilio Frazzoli, Daniel Morton, and Marco Pavone. Toward a Systematic Approach to the Design and Evaluation of Automated Mobility-on-Demand Systems: A Case Study in Singapore. Road Vehicle Automation (Lecture Notes in Mobility), April 2014.

[9] Shared use Mobility Center. Shared-use Mobility - Reference Guide. Technical report, Shared-use Mobility Center, October 2016.

[10] Rick Zhang and Marco Pavone. Control of Robotic Mobility-ondemand Systems. Int. J. Rob. Res., 35(1-3):186-203, January 2016.

[11] Shirley Zhu and Alain Kornhauser. The Interplay Between Fleet Size, Level-of-Service and Empty Vehicle Repositioning Strategies in LargeScale, Shared-Ride Autonomous Taxi Mobility-on-Demand Scenarios. In Transportation Research Board 96th Annual Meeting, 2017.

[12] Tong Zhu. Toward a systematic approach to the fleet size estimation of autonomous mobility-on-demand systems. Msc Thesis, Cornell University, May 2017.

\section{APPENDIX}

Proof of Proposition 2.

Proof. Observe that for a particular $o, d \in X$, it holds that

$$
\sum_{m \in \mathbb{M}} p_{T_{o d}^{-1}(\mathbf{m})}(t)=\sum_{m \in \mathbb{M}^{o>1}, d<c_{d}} p_{\mathbf{m}}(t)
$$

and consequently we also have

$$
\begin{aligned}
\sum_{m \in \mathbb{M}} \sum_{o, d \in X} \lambda_{o d}(t) \cdot p_{T_{o d}^{-1}(\mathbf{m})}(t) & \\
= & \sum_{m \in \mathbb{M}^{o>1}, d<c_{d}} \sum_{o, d \in X} \lambda_{o d}(t) p_{\mathbf{m}}(t) .
\end{aligned}
$$

The sum $\sum_{m \in \mathbb{M}} p_{\mathbf{m}}(t)+p_{F}(t)$ is preserved over every discontinuity $t \in \rho$. To see that

$$
\sum_{m \in \mathbb{M}} p_{\mathbf{m}}(t)+p_{F}(t)=\lim _{t^{\prime} \rightarrow t^{-}} \sum_{m \in \mathbb{M}} p_{\mathbf{m}}\left(t^{\prime}\right)+p_{F}\left(t^{\prime}\right)
$$

holds for any $t \in \rho$, take arbitrary $o, d \in X$ and $t \in \rho_{\text {od }}$ and perform the following rearrangement

$$
\begin{gathered}
\sum_{m \in \mathbb{M}} p_{\mathbf{m}}(t)+p_{F}(t) \\
=\sum_{m \in \mathbb{M}} \lim _{t^{\prime} \rightarrow t^{-}} p_{T_{o d}^{-1}(\mathbf{m})}\left(t^{\prime}\right) \\
+\lim _{t^{\prime} \rightarrow t^{-}} p_{F}\left(t^{\prime}\right)+\sum_{\mathbf{m} \in \mathbb{M}^{o=0} \cup \mathbb{M}^{d=c_{i}}} p_{\mathbf{m}}\left(t^{\prime}\right) \\
=\lim _{t^{\prime} \rightarrow t^{-}} \sum_{m \in \mathbb{M}^{o>1}, d<c_{d}} p_{\mathbf{m}}\left(t^{\prime}\right)+p_{F}\left(t^{\prime}\right)+\sum_{\mathbf{m} \in \mathbb{M}^{o=0} \cup \mathbb{M}^{d=c_{i}}} p_{\mathbf{m}}\left(t^{\prime}\right) \\
=\lim _{t^{\prime} \rightarrow t^{-}} \sum_{m \in \mathbb{M}} p_{\mathbf{m}}\left(t^{\prime}\right)+p_{F}\left(t^{\prime}\right) .
\end{gathered}
$$

Further, we have $\sum_{m \in \mathbb{M}} \dot{p}_{\mathbf{m}}(t)-\dot{p}_{F}(t)=0$ at all $t \in$ $\mathbb{R}_{\geq 0} \backslash \rho$ by the following rearrangement

$$
\begin{aligned}
\sum_{m \in \mathbb{M}} \dot{p}_{\mathbf{m}}(t) & =\sum_{o, d \in X} \lambda_{o d}(t)\left(\sum_{m \in \mathbb{M}^{o>1, d<c_{d}}} p_{\mathbf{m}}(t)-\sum_{m \in \mathbb{M}} p_{\mathbf{m}}(t)\right) \\
& =-\sum_{o, d \in X} \lambda_{o d}(t) \sum_{\mathbf{m} \in \mathbb{M}^{o=0} \cup \mathbb{M}^{d=c_{i}}} p_{\mathbf{m}}(t) \\
& =-\dot{p}_{F}(t),
\end{aligned}
$$

and thus the sum $\sum_{m \in \mathbb{M}} p_{\mathbf{m}}(t)+p_{F}(t)$ is also preserved over every interval where functions $\left\{p_{\mathbf{m}}\right\}_{\mathbf{m} \in \mathbb{M}}$ and $p_{F}$ are differentiable.

We have $\sum_{m \in \mathbb{M}} p_{\mathbf{m}}(0)+p_{F}(0)=1$ at $t=0$ from the initial conditions and since $\sum_{m \in \mathbb{M}} p_{\mathbf{m}}(t)+p_{F}(t)$ is constant for all $t>0$, we conclude that $\sum_{m \in \mathbb{M}} p_{\mathbf{m}}(t)+p_{F}(t)=1$ for all $t \geq 0$.

\section{Proof of Theorem 3 .}

Proof. Let $s_{i}^{j}(t):=P\left(V_{i}(t)\right)=\sum_{\mathbf{m} \in \mathbb{M}^{i=j}} p_{\mathbf{m}}(t)$. Define

$$
X_{o d}^{i=j}(t):=\sum_{\mathbf{m} \in \mathbb{M}^{i=j}} p_{T_{o d}^{-1}(\mathbf{m})}(t)
$$

$$
= \begin{cases}\sum_{\mathbf{m} \in \mathbb{M}^{i=j, o>0, d<c_{d}}} p_{\mathbf{m}}(t) & \text { if } o \neq i \text { and } d \neq i \\ \sum_{\mathbf{m} \in \mathbb{M}^{i=j-1, o>0}} p_{\mathbf{m}}(t) & \text { if } d=i \text { and } j>1 \\ \sum_{\mathbf{m} \in \mathbb{M}^{i=j+1, d<c_{d}}} p_{\mathbf{m}}(t) & \text { if } o=i \text { and } j<c_{d} \\ 0 & \text { otherwise. }\end{cases}
$$

$= \begin{cases}s_{i}^{j}(t)-\sum_{\mathbf{m} \in \mathbb{M}^{i=j, o=0} \cup \mathbb{M}^{i=j, d=c_{d}}} p_{\mathbf{m}}(t) & \text { if } o \neq i \text { and } d \neq i \\ s_{i}^{j-1}(t)-\sum_{\mathbf{m} \in \mathbb{M}^{i=j-1, o=0} p_{\mathbf{m}}(t)} & \text { if } d=i \text { and } j>1 \\ s_{i}^{j+1}(t)-\sum_{\mathbf{m} \in \mathbb{M}^{i=j+1, d=c_{d}} p_{\mathbf{m}}(t)} & \text { if } o=i \text { and } j<c_{d} \\ 0 & \text { otherwise. }\end{cases}$

$$
\begin{gathered}
\text { Let } \alpha_{o d}^{i=j}(t):=\sum_{\mathbf{m} \in \mathbb{M}^{i=j, o=0} \cup \mathbb{M}^{i=j, d=c_{d}}} p_{\mathbf{m}}(t), \beta_{o}^{i=j}(t):= \\
\sum_{\mathbf{m} \in \mathbb{M}^{i=j, o=0}} p_{\mathbf{m}}(t) \text { and } \gamma_{d}^{i=j}(t):=\sum_{\mathbf{m} \in \mathbb{M}^{i=j, d=c_{d}}} p_{\mathbf{m}}(t),
\end{gathered}
$$


which allows us to express $X_{o d}^{i=j}(t)$ more concisely as

$$
X_{o d}^{i=j}(t)= \begin{cases}s_{i}^{j}(t)-\alpha_{o d}^{i=j}(t) & \text { if } o \neq i \text { and } d \neq i \\ s_{i}^{j-1}(t)-\beta_{o}^{i=j}(t) & \text { if } d=i \text { and } j>0 \\ s_{i}^{j+1}(t)-\gamma_{d}^{i=j}(t) & \text { if } o=i \text { and } j<c_{d} \\ 0 & \text { otherwise. }\end{cases}
$$

Function $s_{i}^{j}(t)$ is piecewise differentiable with discontinuous jumps at time points $t_{1}, t_{2}, \ldots \in \rho$. For every $o, d \in X$ and every time point $t \in \rho_{o d}$, we have

$$
\begin{aligned}
s_{i}^{j}(t) & =\sum_{\mathbf{m} \in \mathbb{M}^{i}=j} \lim _{t^{\prime} \rightarrow t} p_{T_{o d}^{-1}(\mathbf{m})}\left(t^{\prime}\right) \\
& =\lim _{t^{\prime} \rightarrow t} X_{o d}^{i=j}\left(t^{\prime}\right) .
\end{aligned}
$$

Using the definition of $s_{i}^{j}$, the derivative of function $s_{i}^{j}$ at every point $t \in \mathbb{R}_{\geq 0} \backslash \rho, \dot{s}_{i}^{j}(t)=$

$$
\sum_{\mathbf{m} \in \mathbb{M}^{i}=j}\left(\sum_{o, d \in X} \lambda_{o d}(t) \cdot p_{T_{o d}^{-1}(\mathbf{m})}-p_{\mathbf{m}} \cdot \sum_{o, d \in X} \lambda_{o d}\right),
$$

which can be rearranged as follows

$$
\begin{aligned}
& \dot{s}_{i}^{j}(t)=\sum_{o, d \in X} \lambda_{o d}(t) \sum_{\mathbf{m} \in \mathbb{M}^{i}=j} p_{T_{o d}^{-1}(\mathbf{m})} \\
& -\sum_{o, d \in X} \lambda_{o d}(t) \sum_{\mathbf{m} \in \mathbb{M}^{i=j}} p_{\mathbf{m}} \\
& =\sum_{o, d \in X} \lambda_{o d}(t) \sum_{\mathbf{m} \in \mathbb{M}^{i=j}} p_{T_{o d}^{-1}(\mathbf{m})} \\
& -s_{i}^{j}(t) \cdot \sum_{o, d \in X} \lambda_{o d}(t) \\
& =\sum_{o, d \in X} \lambda_{o d}(t) \cdot X_{o d}^{i=j}(t)-s_{i}^{j}(t) \cdot \sum_{o, d \in X} \lambda_{o d}(t) . \\
& =\sum_{o, d \in X, o \neq i, d \neq i} \lambda_{o d}(t) \cdot\left(s_{i}^{j}(t)-\alpha_{o d}^{i=j}(t)\right) \\
& +\sum_{o \in X} \lambda_{o i}(t) \cdot s_{i}^{j-1}(t)-\lambda_{o i}(t) \cdot \beta_{o}^{i=j-1}(t) \\
& +\sum_{d \in X} \lambda_{i d}(t) \cdot s_{i}^{j+1}(t)-\lambda_{i d}(t) \cdot \gamma_{d}^{i=j+1}(t) \\
& -\sum_{o, d \in X} \lambda_{o d}(t) \cdot s_{i}^{j}(t) \\
& =\lambda_{i}^{a}(t) \cdot s_{i}^{j-1}(t) \\
& -\left(\lambda_{i}^{a}(t)+\lambda_{i}^{d}(t)\right) \cdot s_{i}^{j}(t) \\
& +\lambda_{i}^{d}(t) \cdot s_{i}^{j+1}(t) \\
& -\sum_{o, d \in X, o \neq i, d \neq i} \lambda_{o d}(t) \cdot \alpha_{o d}^{i=j}(t) \\
& -\sum_{o \in X} \lambda_{o i}(t) \cdot \beta_{o}^{i=j-1}(t) \\
& -\sum_{d \in X} \lambda_{i d}(t) \cdot \gamma_{o}^{i=j+1}(t)
\end{aligned}
$$

Recall now the definition of function $q_{i}^{j}$ and let

$$
Y_{o d}^{i=j}(t):= \begin{cases}q_{i}^{j}(t) & \text { if } o \neq i \text { and } d \neq i \\ q_{i}^{j-1}(t) & \text { if } d=i \text { and } j>0 \\ q_{i}^{j+1}(t) & \text { if } o=i \text { and } j<c_{d} \\ 0 & \text { otherwise. }\end{cases}
$$

The functions $q_{i}^{j}$ and $s_{i}^{j}$ are equal at $t=0$ and thus we have $q_{i}^{j}(0)=s_{i}^{j}(0)$. The behavior of $q_{i}^{j}$ at discontinuities is

$$
\begin{aligned}
& q_{i}^{j}(t)=\lim _{t^{\prime} \rightarrow t^{-}} q_{i}^{j-1}\left(t^{\prime}\right) \quad \forall t \in \rho_{i}^{a} \\
& q_{i}^{j}(t)=\lim _{t^{\prime} \rightarrow t^{-}} q_{i}^{j+1}\left(t^{\prime}\right) \quad \forall t \in \rho_{i}^{d}
\end{aligned}
$$

which can be also expressed as: $\forall o, d \in X, \forall t \in \rho_{o d}$,

$$
\begin{aligned}
q_{i}^{j}(t) & = \begin{cases}\lim _{t^{\prime} \rightarrow t^{-}} q_{i}^{j}\left(t^{\prime}\right) & \text { if } o \neq i \text { and } d \neq i \\
\lim _{t^{\prime} \rightarrow t^{-}} q_{i}^{j-1}\left(t^{\prime}\right) & \text { if } d=i \text { and } j>0 \\
\lim _{t^{\prime} \rightarrow t^{-}} q_{i}^{j+1}\left(t^{\prime}\right) & \text { if } o=i \text { and } j<c_{d} \\
0 & \text { otherwise }\end{cases} \\
= & \lim _{t^{\prime} \rightarrow t^{-}} Y_{o d}^{i=j}\left(t^{\prime}\right) .
\end{aligned}
$$

The behavior everywhere else is described as: $\forall t \in \mathbb{R}_{\geq 0} \backslash \rho$,

$$
\begin{aligned}
\dot{q}_{i}^{j}(t)= & \lambda_{i}^{a}(t) \cdot q_{i}^{j-1}(t) \\
& -\left(\lambda_{i}^{a}(t)+\lambda_{i}^{d}(t)\right) \cdot q_{i}^{j}(t) \\
& +\lambda_{i}^{d}(t) \cdot q_{i}^{j+1}(t)
\end{aligned}
$$

The vector versions of functions $s_{i}^{j}$ and $q_{i}^{j}$ are denoted as

$$
\begin{aligned}
\mathbf{s}_{i}(t) & :=\left[s_{i}^{1}(t), \ldots, s_{i}^{c_{i}}(t)\right] \\
\mathbf{q}_{i}(t) & :=\left[q_{i}^{1}(t), \ldots, q_{i}^{c_{i}}(t)\right] .
\end{aligned}
$$

We will now analyze the behavior of the functions when they are differentiable. Let $\left(t_{s}, t_{e}\right)$ be an subinterval of positive real line satisfying $\left(t_{s}, t_{e}\right) \cap \rho=\emptyset$. Then, the evolution of both vector function $\mathbf{s}_{i}$ and $\mathbf{q}_{i}$ can be described in a form of matrix differential equation with identical time-dependent coefficient matrix $Q_{i}$ :

$$
\begin{aligned}
\mathbf{s}_{i}^{\prime}(t) & :=Q_{i}(t) \cdot s_{i}(t)+\mathbf{b}_{i}(t) \\
\mathbf{q}_{i}^{\prime}(t) & :=Q_{i}(t) \cdot q_{i}(t),
\end{aligned}
$$

where $Q_{i}(t):=$

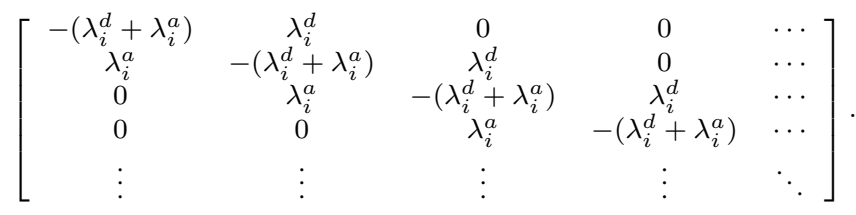

(the argument tof functions $\lambda_{i}^{d}$ and $\lambda_{i}^{a}$ was dropped for brevity)

and $\mathbf{b}_{i}(t)=\left[b_{i}^{1}(t), \ldots, b_{i}^{c_{i}}(t)\right]$ with $b_{i}^{j}(t):=$

$$
\begin{gathered}
-\sum_{o, d \in X, o \neq i, d \neq i} \lambda_{o d}(t) \cdot \alpha_{o d}^{i=j}(t) \\
-\sum_{o \in X} \lambda_{o i}(t) \cdot \beta_{o}^{i=j-1}(t) \\
-\sum_{d \in X} \lambda_{i d}(t) \cdot \gamma_{o}^{i=j+1}(t)
\end{gathered}
$$


Note that we have $\mathbf{b}_{i}^{j}(t) \leq 0 \forall i, j, t$. The solution to the homogenous differential equation (4) have the form $\mathbf{q}_{i}(t)=\Phi\left(t, t_{s}\right) \mathbf{q}_{i}\left(t_{s}\right), \Phi\left(t, t_{s}\right)=\mathcal{T} e^{\int_{t_{s}}^{t} Q(\tau) d \tau}$, where $\mathcal{T}$ is the time ordering operator, taking care of the non-commutativity of $Q$ at different times. Similarly, the solution to the non-homogenous counterpart (3) is $\mathbf{s}_{i}(t)=\Phi\left(t, t_{s}\right) \mathbf{s}_{i}\left(t_{s}\right)+\Phi\left(t, t_{s}\right) \int_{t_{s}}^{t} \Phi\left(t^{\prime}, t_{s}\right)^{-1} \mathbf{b}_{i}\left(t^{\prime}\right) d t^{\prime}=$ $\Phi\left(t, t_{s}\right) \mathbf{s}_{i}\left(t_{s}\right)+\int_{t_{s}}^{t} \Phi\left(t, t^{\prime}\right) \mathbf{b}_{i}\left(t^{\prime}\right) d t^{\prime}$. The matrix $\Phi\left(t, t^{\prime}\right)$ in the integrand is the same that governs the time evolution of the probabilities $\mathbf{q}_{i}(t)$ in the homogeneus solution, hence it maps non-negative vectors to non-negative vectors. The solution $\mathbf{s}_{i}$ is a sum of two terms. Notice that when $\mathbf{s}_{i}\left(t_{s}\right) \leq$ $\mathbf{q}_{i}\left(t_{s}\right)$, then at any time $t>t_{s}$, the first term has lower or equal value than $\mathbf{q}_{i}$. Further, since $\mathbf{b}_{i}^{j}(t) \leq 0$, the second term is bound to be zero or negative. Consequently, we have

$$
\begin{gathered}
\forall t \in\left(t_{s}, t_{e}\right), t_{s}, t_{e} \in \mathbb{R}_{\geq 0}, \quad\left(t_{s}, t_{e}\right) \cap \rho=\emptyset: \\
\quad \text { if } \mathbf{s}_{i}\left(t_{s}\right) \leq \mathbf{q}_{i}\left(t_{s}\right), \text { then } \mathbf{s}_{i}(t) \leq \mathbf{q}_{i}(t) .
\end{gathered}
$$

We will now generalize the above result to the entire time domain. The deterministic rebalancing partitions the time domain into time points $\tau_{1}, \tau_{2}, \tau_{3}, \ldots \in \rho$ where the functions $\mathbf{s}_{i}$ and $\mathbf{q}_{i}$ are discontinuous and intervals $\left(\tau_{0}=0, \tau_{1}\right),\left(\tau_{1}, \tau_{2}\right),\left(\tau_{2}, \tau_{3}\right), \ldots$ on which $\mathbf{s}_{i}$ and $\mathbf{q}_{i}$ are differentiable. We will show that $\mathbf{s}_{i}\left(t^{\prime}\right) \leq \mathbf{q}_{i}\left(t^{\prime}\right) \forall t^{\prime} \in \mathbb{R}_{\geq 0}$ by induction over such differentiable intervals.

Base step: We have $\mathbf{s}_{i}(0)=\mathbf{q}_{i}(0)$ and thus $\mathbf{s}_{i}\left(\tau_{0}\right) \leq \mathbf{q}_{i}\left(\tau_{0}\right)$ holds.

Induction step: Assume $\mathbf{s}_{i}\left(\tau_{i-1}\right) \leq \mathbf{q}_{i}\left(\tau_{i-1}\right)$. Using the property 5e know that $\mathbf{s}_{i}(t) \leq \mathbf{q}_{i}(t) \forall t \in\left[\tau_{i-1}, \tau_{i}\right)$. Observe that if $\mathbf{s}_{i}(t) \leq \mathbf{q}_{i}(t)$, then $X_{o d}^{i=j}(t) \leq Y_{o d}^{i=j}(t) \forall i, j, o, d$ and consequently, we have $\mathbf{s}_{i}\left(\tau_{i}\right) \leq \mathbf{q}_{i}\left(\tau_{i}\right)$.

Finally recall that by definition $s_{i}^{j}(t)=P\left(V_{i}(t)=j\right)$ and $q_{i}^{j}(t)=P\left(\bar{V}_{i}(t)=j\right)$ and thus we have $P\left(V_{i}(t)=j\right) \leq$ $P\left(\bar{V}_{i}(t)=j\right)$ for all $i \in X, j \in \mathbb{M}_{i}$, and $t \geq 0$.

\section{Proof of Corollary 4 .}

Proof. Recall that

$$
\dot{p}_{F}(t)=\sum_{o, d \in X} \lambda_{o d}(t) \sum_{\mathbf{m} \in \mathbb{M}^{o=0} \cup \mathbb{M}^{d=c_{i}}} p_{\mathbf{m}}(t) .
$$

Observe that for particular $o, d \in X$, it holds that

$$
\sum_{\mathbf{m} \in \mathbb{M}^{o=0} \cup \mathbb{M}^{d=c_{i}}} p_{\mathbf{m}}(t) \leq \sum_{\mathbf{m} \in \mathbb{M}^{o=0}} p_{\mathbf{m}}(t)+\sum_{\mathbf{m} \in \mathbb{M}^{d=c_{d}}} p_{\mathbf{m}}(t) .
$$

Consequently, we have

$\dot{p}_{F}(t) \leq \sum_{o, d \in X} \lambda_{o d}(t)\left(\sum_{\mathbf{m} \in \mathbb{M}^{o}=0} p_{\mathbf{m}}(t)+\sum_{\mathbf{m} \in \mathbb{M}^{d=c_{d}}} p_{\mathbf{m}}(t)\right)$

which implies

$$
\begin{aligned}
\dot{p}_{F}(t) \leq & \sum_{o, d \in X} \lambda_{o d}(t) \sum_{\mathbf{m} \in \mathbb{M}^{o=0}} p_{\mathbf{m}}(t) \\
& +\sum_{o, d \in X} \lambda_{o d}(t) \sum_{\mathbf{m} \in \mathbb{M}^{d=c_{d}}} p_{\mathbf{m}}(t) .
\end{aligned}
$$

From Theorem 3 , we know $\sum_{\mathbf{m} \in \mathbb{M}^{i=j}} p_{\mathbf{m}}(t) \leq q_{i}^{j}(t)$ and thus

$$
\begin{aligned}
& \dot{p}_{F}(t) \leq \sum_{o, d \in X} \lambda_{o d}(t) q_{o}^{0}(t)+\sum_{o, d \in X} \lambda_{o d}(t) q_{d}^{c_{d}}(t) \\
& \dot{p}_{F}(t) \leq \sum_{o \in X} q_{o}^{0}(t) \sum_{d \in X} \lambda_{o d}(t)+\sum_{d \in X} q_{d}^{c_{d}}(t) \sum_{d \in X} \lambda_{o d}(t) \\
& \dot{p}_{F}(t) \leq \sum_{i \in X}\left(\lambda_{i}^{d}(t) q_{i}^{0}(t)+\lambda_{i}^{a}(t) q_{d}^{c_{d}}(t)\right) \\
& \dot{p}_{F}(t) \leq \sum_{i \in X} \dot{q}_{i}^{F}(t) .
\end{aligned}
$$

From the above upper bound on $\dot{p}_{F}(t)$ and using $p_{F}(0)=0$ and $q_{i}^{F}(0)=0 \forall i \in X$, we conclude $p_{F}(t) \leq \sum_{i \in X} q_{i}^{F}(t)$. 